\title{
Dynamic behavior of the monomer-monomer surface reaction model with adsorbate interactions
}

\author{
Christopher A. Voigt and Robert M. Ziff \\ Department of Chemical Engineering, University of Michigan, Ann Arbor, Michigan 48109-2136
}

(Received 13 March 1997; accepted 1 August 1997)

\begin{abstract}
The monomer-monomer surface reaction model with an adsorbate interaction term is studied. An epidemic analysis of the poisoning times $\left(t_{p}\right)$ for small square lattices as a function of lattice edge length $L$ and interaction strength $\alpha$ at the point of equal adsorption rates yields a dynamic scaling relation which describes the crossover between log-power-law and exponential behavior in $L$, and is able to fit the entire dependence of $t_{p}$ upon $\alpha$ and $L$. The phase transition is further explored by varying adsorption rates and is found to follow second-order kinetics. A mean-field approximation is introduced as a comparison for the numerical results. (c) 1997 American Institute of Physics.
\end{abstract} [S0021-9606(97)00342-5]

\section{INTRODUCTION}

Lattice models have been used to successfully predict a wide range of experimental observations in catalysis. ${ }^{1}$ The relevance of simulating catalytic behavior at the atomic level leads to a better understanding of the phenomena observed to effect the kinetics of macroscopic applications. Numerous papers have been written dealing with the theoretical simulation of catalytic problems. ${ }^{2}$ In the Ziff-Gulari-Barshad (ZGB) model, the simulation of $\mathrm{CO}(\mathrm{A})$ and $\mathrm{O}_{2}\left(\mathrm{~B}_{2}\right)$ on a $\mathrm{Pt}$ surface was studied using a simplified three-step model representing the Langmuir-Hinschelwood process. ${ }^{3}$ This model displays kinetic phase transitions dependent on the probability for $A$ adsorption $(p)$ vs $B$ adsorption $(1-p)$. When $p$ is increased beyond $p_{2}$ or decreased below $p_{1}$, the system becomes saturated with a single species. This process, often referred to as poisoning, has been confirmed with experimental data for the CO transition. ${ }^{1,4,5}$ For $p$ between $p_{1}$ and $p_{2}$, the system exists in an effectively reactive steady state. However, for a finite-size system, this reactive steady state is technically metastable, since the reaction will stop by a fluctuation if the system becomes covered by any species. This phenomenon can only be seen for very small systems because the time to poison grows exponentially with the size of the system. ${ }^{6}$

In this paper, we study the simpler monomer-monomer $(A B)$ model, first studied by Wicke et al. ${ }^{7}$ The model is based upon the generic three-step mechanism:

$$
\begin{aligned}
& A{ }^{*} \rightarrow A^{*}, \\
& B+* \rightarrow B^{*}, \\
& A^{*}+B^{*} \rightarrow A B+2^{*},
\end{aligned}
$$

where * refers to a lattice site. The model also contains a single parameter $p_{A}$ which gives the relative probability that an $A$ molecule strikes the surface, so that the probability that a $B$ strikes the surface is $1-p_{A}$. When the adsorption probabilities are held constant at any value, the system always poisons. When $p_{A} \neq 1 / 2$, it poisons relatively quickly with $A(p>1 / 2)$ or $B(p<1 / 2)$ particles. When $p_{A}=1 / 2$, poisoning is still found to occur, but at a much slower rate. For times beyond the poisoning time $t_{p}$, the probability of the system not being poisoned has been found to decrease exponentially with time. ${ }^{8}$

Here, we attempt to avert poisoning by introducing small energetic interactions between the molecules in such a way as to cause a feedback mechanism between the concentration of molecules adsorbed on the surface and the rate of adsorption. Such a mechanism should interfere with the simple statistical poisoning mechanism described above. A similar effect was qualitatively studied in the work of Moiny et al. ${ }^{9}$ where a minimum value of the interaction strength was found to be necessary in order to stop the poisoning of the system for a $100 \times 100$ lattice (their result $D$ ). Frachebourg et al. studied the effect of quenched randomness on the $A B$ model surface and found a rich variety of kinetic effects. ${ }^{10}$ The disorder caused a phase transition similar to ours which could also be predicted using a mean-field analysis. The effects of similar interactions on the ZGB model have also been studied, ${ }^{11}$ where it was found that, by increasing the repulsive nature of $A$ and $B$, the reactive window widens and the phase transitions become smoother. Because the model that we explore is based on a few fundamental mechanisms, there are a wide variety of physical systems for which its dynamics may be relevant.

\section{THE MODEL}

We consider the adsorption-limited $A B$ model, in which the rate of reaction is assumed to be so much greater than the rate of adsorption that the lattice never contains any adjacent $A B$ pairs (as in the original ZGB model) and the reactions are carried out instantaneously. The other limiting case, where the reaction is the limiting step, has also been considered. ${ }^{12-14}$ For each Monte Carlo step, a site is randomly chosen. If that site is empty, an $A$ or $B$ particle is adsorbed with the local probabilities as described below. A Monte Carlo time step corresponds to the number of adsorption attempts equaling the number of sites in the lattice. The reaction is assumed to occur between the species being adsorbed and any of its eight nearest and next-nearest neighbors with equal probability. We assume that there is no spon- 

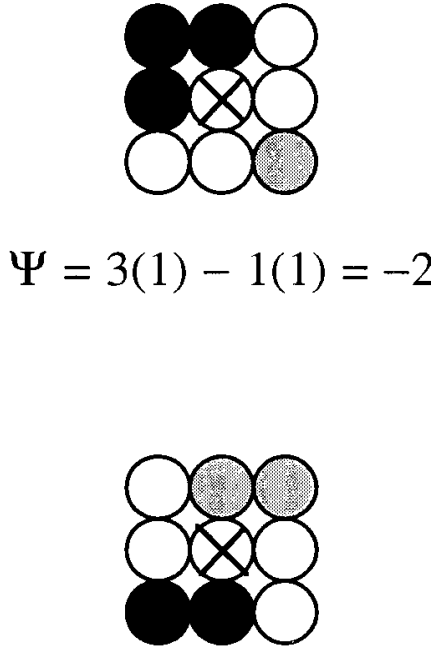

$\Psi=2(1)-2(1)=0$

FIG. 1. Example of the determination of interparticle energy. The black circles are $A$, the gray are $B$, and the while are vacant spaces. The " $\mathrm{x}$ " is the empty space being analyzed.

taneous desorption of unreacted $A$ or $B$ molecules, nor any diffusion of adsorbed species. Periodic boundary conditions were applied.

Unlike the previous studies of the $A B$ model, the probabilities for adsorption were not fixed throughout this simulation. Rather, an evaluation was conducted about the adsorption site during the Monte Carlo process to determine the probability a species will adsorb at that specific point. It is set that like molecules exhibit repulsive behavior while unlike ones attract. The interaction energy $(\Psi)$ of a lattice site was assumed to be proportional to the number of $A$ minus $B$ neighbors and next-nearest neighbors about the given site, as shown in Fig. 1,

$$
\Psi=N_{A}-N_{B} .
$$

The range of $\Psi$ is therefore $(-8,8)$ and is independent of system clustering. The probability to adsorb an $A$ was then calculated from

$$
p_{A}=p_{0}-\alpha \Psi,
$$

where $\alpha$ is a measure of the interaction strength, and is a function of temperature and reaction rate for actual catalytic systems. The value $p_{0}$ is related to the relative rate that $A$ molecules strike the surface. Most of our simulations are done at $p_{0}=0.5$. The conditions $\alpha=0$ and $p_{A}=p_{0}$ represent the hard-sphere limit of the system. Equation (5) represents a linearization of any typical thermally activated rate, such as $p_{A}=e^{-\alpha \Psi} /\left(e^{\alpha \Psi}+e^{-\alpha \Psi}\right)$, for small $\alpha$. Because $\alpha \Psi$ $\propto E / k T$, we are assuming that $E / k T \ll 1$. Although $\alpha \Psi$ is small enough to justify the linearization, it leads to a large modification of the behavior.

The magnitude of $\alpha$ was found to have a strong effect on the configuration of surface species. As $\alpha$ was increased, clusters of homogeneous particles became smaller, making

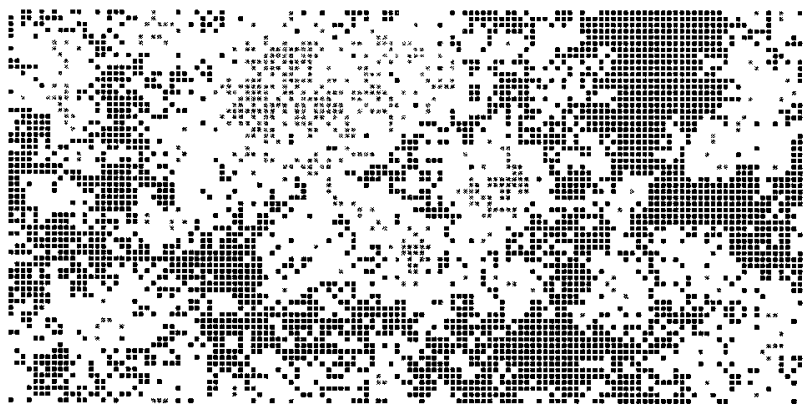

(a)

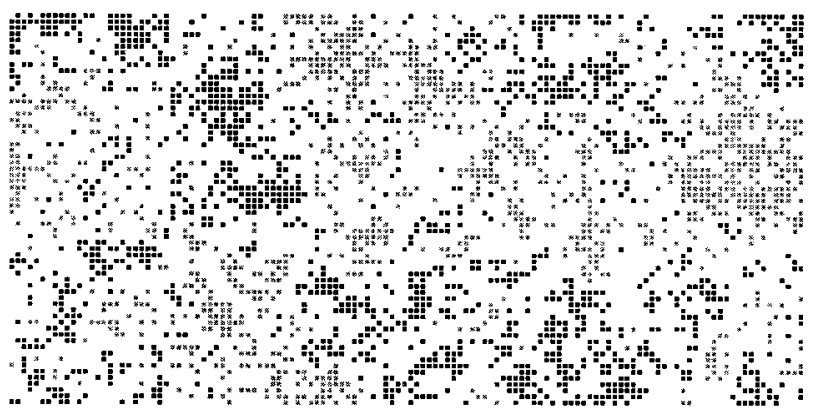

(b)

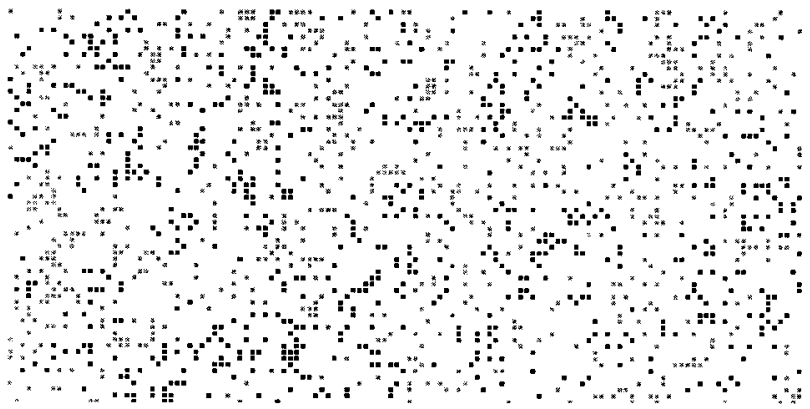

(c)

FIG. 2. Observations of the effect of increasing energetic interactions on a $64 \times 128$ matrix, for $\alpha=0$ (a), 0.00125 (b), and 0.03125 (c). These snapshots were taken after 1024 Monte Carlo time steps. Black dots represent $A$ molecules, grey represent $B$, and vacant sites are white.

the environment less likely to saturate. This behavior is shown in Figs. 2(a) and 2(b). Figure 2(c) displays the behavior of a system with high $\alpha$ in which there is essentially no clustering. The interface between domains has all but disappeared. As the clusters shrink, reactions only occur around their boundaries, so the overall surface reaction rate increases with $\alpha$. $^{15,16}$

\section{MONTE CARLO SIMULATION RESULTS}

An epidemiclike analysis ${ }^{17-20}$ was run for various square lattice edge lengths $(L)$ and $\alpha$ to find the number of Monte Carlo time steps it took for the system to poison $\left(t_{p}\right)$. For each combination of $L$ and $\alpha, 400$ trials were taken and the results were averaged. The simulation was run for values of $\alpha$ between 0 and 0.03125 in increments of 0.0003125 , although most systems reached steady state well before $\alpha$ 


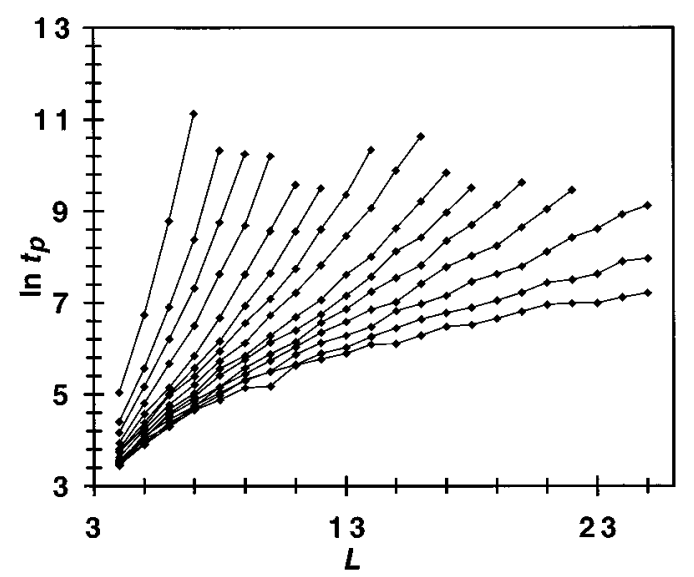

FIG. 3. Plot of $\ln t_{p}$ vs $L$ with increasing $\alpha$. Values of $\alpha$ from bottom to top: $0,0.000313,0.000625,0.000938,0.00125,0.001563,0.001875$, $0.0025,0.003125,0.00375,0.004688,0.00625,0.007812,0.01$, 0.014375 .

reached its maximum value. Lattice sizes studied ranged from $4 \times 4$ to $25 \times 25$. The parameter $p_{0}$ was fixed at 0.5 for these runs.

The results of the simulation are shown in Fig. 3. When $\alpha \neq 0, t_{p}$ grows exponentially with $L$, implying that large systems will effectively reach a reactive steady state. For $\alpha=0$, the $A B$ model has been solved exactly, and the saturation time has been shown to have a linear dependence on the number of catalyst sites with logarithmic corrections: $:^{14,21}$

$$
t_{p} \propto L^{2}(1+c \ln L) \text {. }
$$

Our data follow this relation and suggest $c \approx 0.16$. Here, poisoning occurs by a simple statistical process. Because there is no feedback mechanism between the coverage on the surface and the adsorption process, and a reaction removes exactly one $A$ and one $B$, the difference between the number of $A$ and $B$ molecules adsorbed on the surface at any given time will equal the difference in the number of $A$ and $B$ molecules that have struck empty sites on the surface for the duration of the simulation. The system will be poisoned when that difference equals the number of sites on the surface, $L^{2}$, and this will occur when the number of successful adsorption trials is of order $L^{4}$, since the fluctuations grow as the square root of the number of trials. If a constant fraction of adsorption trials were successful, then Monte Carlo time would be proportional to $L^{2}$, and $t_{p}$ would grow exactly as $L^{2}$. However, as time progresses, the fraction of successful adsorption trials decreases, and because time is measured in terms of successful as well as unsuccessful adsorption attempts, the growth is somewhat smaller and leads to the logarithmic correction. ${ }^{14,21}$ This type of logarithmic correction has the practical effect of giving apparent dynamic exponents of about $2.03-2.10$ if a simple power law were (erroneously) assumed, as indeed we had done previously, before the exact solution was known. ${ }^{22}$

For small values of $\alpha$, there is a transitional region as the log-power-law relation between $t_{p}$ and $L$ evolves into an exponential relation as $L$ increases. This transition is a

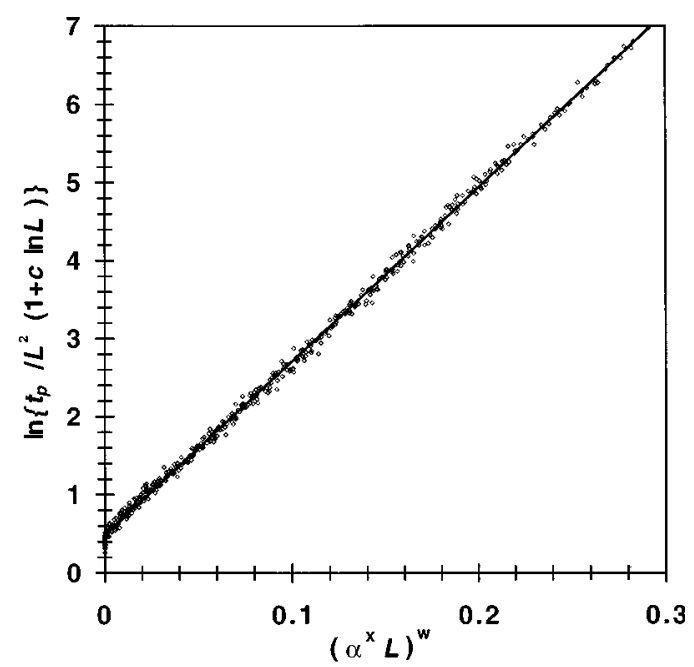

FIG. 4. Collapse of data of Fig. 3 using the scaling relation (7), with $x$ $=0.575, z=0.16$, and $w=2.50$. The slope of this line was found to be 22.2 and the intercept 0.489 .

gradual process and the time it occurs decreases as $\alpha$ increases. The transitional region was never observed to disappear completely for large $\alpha$.

In the exponentially growing region, $t_{p}$ was found to follow behavior of the form:

$$
t_{p} \propto e^{\left(\alpha^{x} L\right)^{w}} \text {. }
$$

This was found by plotting $\ln t_{p}$ vs $L^{w}$ for different $w$ until straight lines were obtained. The final values of $x$ and $w$ were found through the scaling plot in Fig. 4. Now, Eq. (6) is valid for small $\alpha$, while (7) is valid for $\alpha \neq 0$ and large $L$. Together, these two equations suggest a general scaling relation of the form

$$
t_{p}=a L^{2}(1+c \ln L) f\left(\alpha^{x} L\right),
$$

where

$$
f(\xi)=a e^{b \xi^{w}}
$$

or, equivalently

$$
t_{p}=a L^{2}(1+c \ln L) e^{b\left(\alpha^{x} L\right)^{w}} .
$$

The validity of this relation is demonstrated in Fig. 4, where we plot $\ln \left[t_{p} / L^{2}(1+c \ln L)\right]$ vs $\left(\alpha^{x} L\right)^{w}$, using values $x$ $=0.575, c=0.16$, and $w=2.50$. With these values of the parameters, it can be seen that all the data collapse to a single straight line, showing that the scaling is valid, and furthermore that the scaling function is a simple exponential as in (9). Remarkably, this simple exponential behavior appears to describe the scaling function over its entire range down to the smallest lattices that we studied. From the slope and intercept of that line we find $a=22.2$ and $b=1.63$. Equation (10) provides a comprehensive model for the scaling behavior of this system, with respect to both $\alpha$ and $L$, that describes the exponential, log-power-law, and transitional regions. Equation (10) predicts that whenever $\alpha$ is not equal to zero, the poisoning time will eventually grow exponentially (for large enough $L$ ), which indicates a reactive steady state. 


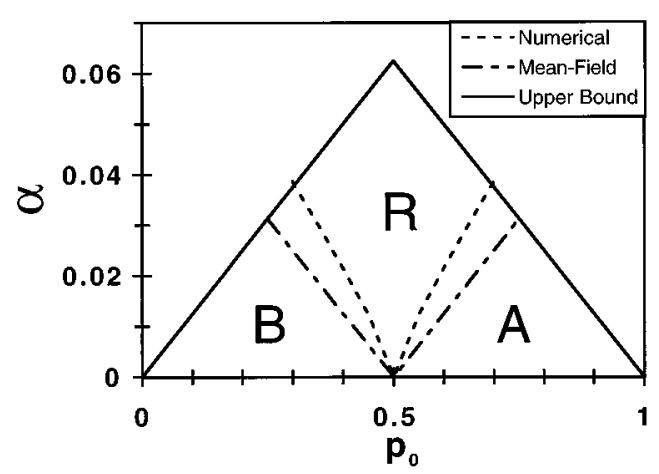

FIG. 5. Phase diagram of $p_{0}$ vs $\alpha$ showing a reactive region as well as saturation regions of $A$ and $B$ on a $25 \times 25$ lattice. The dotted line represents the numerical data and the solid line is the mean-field analysis. The letters indicate the phases. The upper bound represents the maximum value of $\alpha$ such that $p_{A}$ falls in the range $(0,1)$.

We also studied the system for $p_{0} \neq 0.5$. Simulations were performed on a $25 \times 25$ lattice and $\alpha$ was increased until a reactive steady state was found. This led to the phase diagram shown in Fig. 5 (dotted line). Note that, $\alpha \neq 0$, a window in $p_{0}$ opens up where the system reaches a reactive steady state.

The order of this transition was determined by lowering $\alpha$ for a fixed $p_{0} \neq 0.5$ and watching the behavior as poisoning occurred. During this process, no islands of a single species were observed to form. The system became slowly saturated throughout the lattice with no sign of significant (effective) surface tension between the species. Furthermore, the coverages of the two species appeared to go continuously to 1 or 0 . This behavior strongly suggests that the phase transition is second order.

\section{MEAN-FIELD ANALYSIS}

To further explore the kinetics of the phase transition, we constructed a mean-field model of the system. ${ }^{23-26}$ Define $X_{A}, X_{B}$, and $X_{V}$ as the coverages of $A, B$, and vacant sites, respectively, with $X_{V}=1-X_{A}-X_{B}$. The single-site rate equations for this model are

$$
\frac{d X_{A}}{d t}=\left\langle p_{A}\right\rangle X_{V}\left(1-X_{B}\right)^{8}-\left(1-\left\langle p_{A}\right\rangle\right) X_{V}\left(1-\left(1-X_{A}\right)^{8}\right),
$$

$$
\frac{d X_{B}}{d t}=\left(1-\left\langle p_{A}\right\rangle\right) X_{V}\left(1-X_{A}\right)^{8}-\left\langle p_{A}\right\rangle X_{V}\left(1-\left(1-X_{B}\right)^{8}\right),
$$

where $\left\langle p_{A}\right\rangle$ represents the average value of $p_{A}$. The first term in (11) represents the increase in $X_{A}$ due to an $A$ adsorbing on a vacant site with no $B$ neighbors. The second term represents the decrease in $X_{A}$ due to a $B$ adsorbing on a vacant site with at least one $A$ neighbor and reacting. Equation (12) is similarly constructed. Subtracting these two equations produces

$$
\frac{d\left(X_{A}-X_{B}\right)}{d t}=X_{V}\left(2\left\langle p_{A}\right\rangle-1\right),
$$

which will go to zero at the phase transition between a reactive and poisoned state. Statistically, it follows from the mean-field hypothesis that

$$
\left\langle N_{A}-N_{B}\right\rangle=8\left(X_{A}-X_{B}\right) .
$$

Combined with equations (4) and (5), this implies

$$
\left\langle p_{A}\right\rangle=p_{0}-8 \alpha\left(X_{A}-X_{B}\right) .
$$

Equations (11), (12), and (13) remain consistent that when $\alpha \rightarrow 0$, the system poisons rather quickly when $p_{0} \neq 0.5$, and remains static otherwise. Now, when the system poisons with $A,\left(X_{A}-X_{B}\right) \rightarrow 1$, and when it poisons with $B,\left(X_{A}\right.$ $\left.-X_{B}\right) \rightarrow-1$. Then, setting (13) to zero yields

$$
\alpha=\left\{\begin{array}{cc}
1 / 8 p_{0}-1 / 16 & \left(p_{0}<0.5\right) \\
-1 / 8 p_{0}+1 / 16 & \left(p_{0}>0.5\right) \\
0 & \left(p_{0}=0.5\right) .
\end{array}\right.
$$

This set is plotted in Fig. 5, along with numerical results that were described in Sec. III. The discrepancy between the simulations and mean-field predictions indicates the importance of long range effects between species in this model, which is not intrinsically considered within the mean-field equations. The transition predicted by (16) is continuous in $X_{A}$ and $X_{B}$, and is therefore second order.

\section{CONCLUSIONS}

A model describing adsorption kinetics involving interspecies interactions has been proposed. Adding a repulsive interaction suppresses the poisoning of the $A B$ model (for a sufficiently large system) and leads to a reactive window in the phase diagram. The phase diagram is qualitatively similar to the phase diagram of the model of Frachebourg et al., where interaction is replaced by quenched surface randomness, which also has the effect of suppressing poisoning. ${ }^{10}$ Along the line $p_{0}=0.5$ and $\alpha \geqslant 0$, we have found a surprisingly simple scaling relation that correlates the poisoning time with lattice size $L$ and interaction parameter $\alpha$. This relation [Eq. (10)] also introduces two new scaling exponents, $x$ and $w$. These results raise the question, as to whether other dynamic surface-reaction models follow a similar, perhaps universal, finite-size poisoning behavior.

\section{ACKNOWLEDGMENT}

This material is based upon work supported by the U. S. National Science Foundation under Grant No. DMR9520700 .

\footnotetext{
${ }^{1}$ R. Imbihl and G. Ertl, Chem. Rev. 95, 697 (1995).

${ }^{2}$ For a review, see E. V. Albano, Heterog. Chem. Rev. 3, 389 (1996).

${ }^{3}$ R. Ziff, E. Gulari, and Y. Barshad, Phys. Rev. Lett. 56, 2553 (1986).

${ }^{4}$ J. Ree, Y. H. Kim, and H. K. Shin, J. Chem. Phys. 104, 742 (1996).

${ }^{5}$ M. Ehsasi, Appl. Surf. Sci. 76, 89 (1994).

${ }^{6}$ P. Meakin and D. J. Scalapino, J. Chem. Phys. 87, 731 (1987).

${ }^{7}$ E. Wicke, P. Kumman, W. Keil, and J. Scheifler, Ber. Bunsenges. Phys. Chem. 4, 315 (1980).

${ }^{8}$ D. ben-Avaham, S. Redner, D. B. Considine, and P. Meakin, J. Phys. A 3, L613 (1990).

${ }^{9}$ F. Moiny, M. Dumont, J. Lion, P. Dufour, and R. Dagonnier, Surf. Sci. Lett. 325, L392 (1995).
} 
${ }^{10}$ L. Frachebourg, P. L. Krapivsky, and S. Redner, Phys. Rev. Lett. 75, 2891 (1995).

${ }^{11}$ J. Mai, V. Kuzovkov, and W. von Niessen, J. Phys. A 29, 6219 (1996).

${ }^{12}$ K. Fichthorn, E. Gulari, and R. Ziff, Phys. Rev. Lett. 63, 1527 (1989).

${ }^{13}$ E. Clément, P. Leroux-Hugon, and L. Sander, J. Stat. Phys. 65, 925 (1991).

${ }^{14}$ P. L. Krapivsky, Phys. Rev. A 45, 1069 (1992).

${ }^{15}$ D. Sholl and R. Skodje, Phys. Rev. E 53, 33 (1996).

${ }^{16}$ A. Datar and D. Prasad, J. Chem. Phys. 100, 1742 (1994).

${ }^{17}$ E. V. Albano, Phys. Rev. Lett. 72, 108 (1994).

${ }^{18}$ H. C. Kang and W. H. Weinberg, J. Chem. Phys. 100, 1630 (1994).
${ }^{19}$ J. W. Evans and M. S. Miesch, Phys. Rev. Lett. 66, 833 (1991).

${ }^{20}$ I. Jensen, H. C. Fogedby, and R. Dickman, Phys. Rev. A 41, 3411 (1990).

${ }^{21}$ E. Clément, P. Leroux-Hugon, and L. Sander, Phys. Rev. Lett. 67, 1661 (1991).

${ }^{22}$ K. Fichthorn, R. Ziff, and E. Gulari, in Catalysis, edited by W. Ward, Proceedings of the North American Catalysis Society, 1987, pp. 883-894.

${ }^{23}$ P. Fischer and U. M. Titulaer, Surf. Sci. 221, 409 (1989).

${ }^{24}$ R. Dickman, Phys. Rev. A 34, 4246 (1986).

${ }^{25}$ V. P. Zhdanov and B. Kasemo, Appl. Surf. Sci. 74, 147 (1994).

${ }^{26}$ J. W. Evans, Langmuir 93, 2514 (1991). 